\title{
TTR
}

Traduction, terminologie, rédaction

\section{Marie-Alice Belle et Álvaro Echeverri, études réunies par. Pour une interdisciplinarité réciproque. Recherches actuelles en traductologie. Arras, Artois Presses Université, 2017, 208 p.}

\section{Céline Letawe}

La politique des microcentres : la traduction dans des contextes " mineurs » comme transfert culturel complexe

The Politics of Micro-Centers: Translation in "Minor" Contexts as Complex Cultural Transfer

Volume 32, numéro 2, 2e semestre 2019

URI : https://id.erudit.org/iderudit/1068911ar

DOI : https://doi.org/10.7202/1068911ar

Aller au sommaire du numéro

Éditeur(s)

Association canadienne de traductologie

ISSN

0835-8443 (imprimé)

1708-2188 (numérique)

Découvrir la revue

Citer ce compte rendu

Letawe, C. (2019). Compte rendu de [Marie-Alice Belle et Álvaro Echeverri, études réunies par. Pour une interdisciplinarité réciproque. Recherches actuelles en traductologie. Arras, Artois Presses Université, 2017, 208 p.] TTR, 32 (2), 247-249. https://doi.org/10.7202/1068911ar d'utilisation que vous pouvez consulter en ligne. 
be. However, its true merit lies in the variety of perspectives it brings together. By combining a theoretical focus with attention to specific artistic, economic, political, and didactic needs, the volume manages to shed new light on a much-discussed subject.

Ellen Lambrechts

KU LeUven

$* * * * * * * * * *$

\section{Marie-Alice Belle et Álvaro Echeverri, études réunies par. Pour une interdisciplinarité réciproque. Recherches actuelles en traductologie. Arras, Artois Presses Université, 2017, 208 p.}

Pour une interdisciplinarité réciproque est le $42^{\mathrm{e}}$ volume de la collection «Traductologie», lancée en 1997 chez Artois Presses Université avec le titre ambitieux et séduisant d'Anthony Pym Pour une éthique $d u$ traducteur. Vingt ans plus tard, la quatrième de couverture des études réunies par Marie-Alice Belle et Álvaro Echeverri promet de «nouvelles pistes théoriques et méthodologiques mettant en valeur la contribution actuelle et potentielle de la [traductologie] aux autres domaines de recherche en sciences humaines». Le volume est né du colloque «Repenser la place de la traductologie dans les sciences humaines : vers une interdisciplinarité réciproque» tenu à Montréal en mai 2012 à l'occasion du 80 congrès de l'Association francophone pour le savoir (Acfas). La décision de remplacer la préposition vers qui figurait dans le titre du colloque par la préposition pour dans le titre du livre traduit la volonté de faire de ce dernier un plaidoyer.

Le constat de Roch Duval, un des contributeurs, est clair : «La traductologie vit des heures difficiles ces derniers temps» (p. 46). En effet, après sêtre définie en tant que discipline en se distinguant de la linguistique d'une part et des études littéraires de l'autre, la traductologie a connu au cours des dernières décennies un «tournant» interdisciplinaire qui a entraîné un véritable boom, mais également un certain flou conceptuel lié à une ouverture toujours plus grande. La discipline a certes profité de nombreuses importations méthodologiques et théoriques (on parlera, avec Klaus Kaindl, d'«interdisciplinarité instrumentale», p. 49) qu'il s'agirait d'approfondir et surtout d'articuler, mais elle n'est hélas pas parvenue à exporter grand-chose (la fameuse interdisciplinarité réciproque du titre, celle qui serait «souhaitable pour consolider la discipline» (p. 25) comme l'indique Nayelli Castro). 
Dans son introduction, Marie-Alice Belle souligne avec perspicacité le besoin de conserver un équilibre entre «le ressourcement théorique et critique que permet le dialogue avec d'autres disciplines» (p. 9) d'une part, et «le maintien d'une certaine identité disciplinaire» de l'autre (ibid.). Toute la tension du livre est résumée dans ces quelques mots.

Le volume est structuré en deux parties. La première est consacrée aux « aspects théoriques et méthodologiques »; la seconde propose cinq illustrations de pratiques "intégrées", c'est-à-dire de recherches en traductologie qui intègrent des apports théoriques et méthodologiques de disciplines diverses (sociologie, études postcoloniales, histoire littéraire et sociale, études littéraires, anthropologie, psychanalyse, théorie littéraire, psychologie cognitive, sciences de l'éducation). La dernière de ces illustrations, "Le discours sur la formation des traducteurs: au-delà des questions linguistiques, ou la quête de la pertinence», est particulièrement intéressante. Incarnant la branche appliquée de la traductologie, Álvaro Echeverri y souligne le «fossé qui sépare les pratiques pédagogiques actuelles de la recherche théorique» (p.13) et encourage le développement d'initiatives de recherche «dans la salle de classe» (p. 172) afin de «valoriser et mettre à profit les connaissances accumulées durant plus de soixante-dix ans de pratique en formation universitaire des traducteurs» (p. 174).

Nous nous concentrerons ici sur la première partie du volume, dont les enjeux et questionnements nous semblent davantage susceptibles d'intéresser tout traductologue, indépendamment de son objet. Dans la première contribution, Nayelli Castro revient sur les métaphores choisies par James Holmes en 1972 pour présenter la traductologie comme une «utopie disciplinaire» (p.20) dans son article fondateur "The Name and Nature of Translation Studies». Castro constate que la traductologie et ses disciplines voisines ont dès le départ été envisagées comme concurrentes et elle propose une nouvelle métaphore, celle du «récit polyphonique» (p. 27), afin «de relativiser le caractère homogène et clos d'une méthodologie et de la considérer comme une narration inachevée ou non définitive» (ibid.).

Dans ce qu'il présente comme un «guide d'orientation» (p. 33), Roch Duval interroge radicalement la méthode traductologique et ses fondements scientifiques. Il va jusqu'à se demander s'il n'y aurait pas «à la base de la traductologie un vice logique, une erreur catégorielle, une méprise conceptuelle» (p.34). Selon lui, le temps est venu de repenser les fondements épistémologiques de la discipline, qui risque sinon de devenir «une sorte de supermarché où on vend à rabais des 
concepts, sans chercher à les préciser davantage » (p. 41). À sa question conclusive "l'interdisciplinarité est-elle la voie de l'avenir?»(p. 47), l'auteur répond par l'affirmative, mais souligne l'importance de trouver un point fixe qui serve de repère. Titulaire d'un double doctorat en traductologie et en philosophie, Duval est convaincu que «seul un discours théorique unitaire - représenté par la philosophie - [...] pourra garantir que les emprunts interdisciplinaires [...] respectent le prérequis qui consiste à asseoir toute science sur une base théorique solide» (p. 50).

Dans la dernière contribution de la première partie du volume, Chantal Gagnon montre à partir de trois études qui analysent des discours politiques sans considérer la traduction ce que les disciplines respectives (psychologie politique, sociologie et science politique) auraient gagné en tenant compte des apports de la traductologie. Son texte est un véritable plaidoyer pour renforcer la collaboration interdisciplinaire, condition sine qua non à une plus grande reconnaissance de la traductologie par les sciences humaines et sociales.

C'est notamment à un travail de conscientisation que ce volume nous invite. Le constat que faisait José Lambert en 2013 dans un précédent numéro de TTR est toujours bien d'actualité :

L'hétérogénéité n'est pas forcément un handicap, mais l'absence de cohésion entre les différentes ailes des TS [Translation Studies] [...] n'est pas de nature à nous rassurer. L'absence d'harmonie, camouflée non sans diplomatie dans le terme TS, ne manque pas de gêner l'intégration avec et dans les autres disciplines. [...] S'il est vrai que les spécialistes de la traduction n'ont pas toujours brillé dans les initiatives interdisciplinaires, la cécité réciproque nest pas rare dans le monde universitaire. (2013, p. 245).

C'est contre cette cécité réciproque qu'il faut lutter. ${ }^{1}$

\section{Références}

Lambert, José (2013). «La sociologie, l'interdisciplinarité et les recherches sur la traduction». TTR, 26, 2, p. 245-268.

Pym, Anthony (1997). Pour une éthique du traducteur. Arras, Artois Presses Université.

\section{Céline Letawe UNIVERSITÉ DE LIÈge}

1. Notons qu'au moment où le présent compte rendu est rédigé (janvier 2020), la revue Syn-Thèses se prépare à sortir un numéro intitulé «Interdisciplinarité et traductologie». 